\title{
Umbilical cord-derived mesenchymal stem cells reversed the suppressive deficiency of $T$ regulatory cells from peripheral blood of patients with multiple sclerosis in a co-culture - a preliminary study
}

\author{
Hongna Yang ${ }^{1,2}$, Jinhua Sun ${ }^{2}$, Feng Wang ${ }^{2}$, Yan $\mathrm{Li}^{2}$, Jianzhong $\mathrm{Bi}^{3}$ and Tingyu $\mathrm{Qu}^{2,4}$ \\ ${ }^{1}$ Department of Critical-Care Medicine, Qilu Hospital of Shandong University, Shandong University, Jinan, Shandong, China \\ 2 Department of Psychiatry, College of Medicine, University of Illinois at Chicago, Chicago, IL, USA \\ ${ }^{3}$ Department of Neurology, The Second Hospital of Shandong University, Shandong University, Jinan, Shandong, China \\ ${ }^{4} \mathrm{R} \& \mathrm{D}$, Cell and Tissue Bank of Shandong Province, Jinan, Shandong, China \\ Correspondence to: Tingyu Qu, email: tqu@uic.edu or tqu2010@hotmail.com \\ Keywords: umbilical cord mesenchymal stem cells, stem cell priming, multiple sclerosis, T regulatory cells, immunomodulation, Im- \\ munology and Microbiology Section, Immune response, Immunity \\ Received: November 21,2015 Accepted: September 12, $2016 \quad$ Published: September 29, 2016
}

\section{ABSTRACT}

The immunoregulatory function of $\mathrm{T}$ regulatory cells (Tregs) is impaired in multiple sclerosis (MS). Recent studies have shown that umbilical cord-derived mesenchymal stem cells (UC-MSCs) exert regulatory effect on the functions of immune cells. Thus, we investigated whether UC-MSCs could improve the impaired function of Tregs from MS patients. Co-cultures of UC-MSCs with PBMCs of MS patients were performed for 3 days. Flow cytometry was used to determine the frequency of Tregs. A cell proliferation assay was used to evaluate the suppressive capacity of Tregs. ELISA was conducted for cytokine analysis in the co-cultures. Our results showed that UC-MSCs significantly increased the frequency of CD4+CD25+CD127low/Tregs in resting $C D 4^{+} T$ cells $(p<0.01)$ from $M S$, accompanied by the significantly augmented production of cytokine prostaglandin E2, transforming growth factor (- $\beta 1$, and interleukin-10, along with a reduced interferon- $Y$ production in these co-cultures $(p<0.05-0.01)$. More importantly, UC-MSC-primed Tregs of MS patients significantly inhibited the proliferation of PHA-stimulated autologous and allogeneic CD4 ${ }^{+} \mathrm{CD}_{25} 5^{-}$ effector cells (Teffs) from MS patients and healthy individuals compared to non-UCMSC-primed (naïve) Tregs from the same MS patients $(p<0.01)$. Furthermore, no remarkable differences in suppressing the proliferation of PHA-stimulated CD4+CD25Teffs was observed in UC-MSC-primed Tregs from MS patients and naïve Tregs from healthy subjects. The impaired suppressive function of Tregs from MS can be completely reversed in a co-culture by UC-MSC modulation. This report is the first to demonstrate that functional defects of Tregs in MS can be repaired in vitro using a simple UC-MSC priming approach.

\section{INTRODUCTION}

Multiple sclerosis (MS) is a chronic inflammatory, demyelinating, and neurodegenerative disorder of the central nervous system (CNS) [1], which affects approximately 400,000 people in the United States and 2.5 million worldwide [2]. Although oral treatment and parenteral administration exist, these treatments are expensive and also have toxic effects. The etiology of MS is currently not clear, evidence now reveals that 
the autoimmune response mediated by the invasion of auto reactive myelin-specific $\mathrm{CD}^{+} \mathrm{T}$ cells is a key pathological feature of MS. Auto-reactive T cells present in MS patients' blood penetrate the blood-brain barrier (BBB) and become active in the central nervous system (CNS) [1]. More importantly, the reduced number and/or function of $\mathrm{T}$ regulatory cells (Tregs) is responsible for the activation of auto-effective $\mathrm{T}$ cells in the peripheral and CNS [3-5].

Tregs play an important role in establishing and maintaining immunologic self-tolerance and immune homeostasis by various mechanisms. These include direct inhibition of auto-reactive $\mathrm{T}$ cell activation by secreting immunosuppressive mediators through cell-to-cell contact and indirectly via inhibition of the stimulatory capacity of antigen-presenting cells (APCs) [6]. Many studies report that the adoptive transfer of autologous or allogenic Tregs reversed or inhibited autoimmune disease development and controlled the allo-immune response to organ and cell transplantation by cell-to-cell contact, secretion of anti-inflammatory cytokines, and modulation of APCs [7-9]. Transplantation of chloroquine-elicited and allogenic Tregs reduced the severity of EAE (experimental autoimmune encephalomyelitis) in an animal model of MS [10]. Pre-clinical and clinical studies have shown that autologous Tregs may have potential as a novel therapy in the treatment of autoimmune diseases. However, studies have confirmed that Tregs, directly from the patients with autoimmune diseases including MS, cannot efficiently inhibit the proliferation of auto-effective $\mathrm{T}$ cells because of their impaired suppressive function $[11,12]$. Therefore, developing clinically applicable protocols that reverse or repair the impaired suppressive function of these Tregs would be necessary before autologous Tregs could be used as real therapeutic cells.

Mesenchymal stem cells (MSCs) as immunomodulatory cells have been receiving attention because MSCs are able to induce FoxP3+ expression in CD4 T cells in vitro and increase the proportion of Tregs in vivo [13-15]. Some groups have reported that the immunosuppressive function of $\mathrm{CD} 4{ }^{+} \mathrm{CD} 25^{+}$Tregs or $\mathrm{CD}^{+} \mathrm{CD} 28^{-}$Tregs from PBMCs of healthy donors are enhanced in vitro by co-culture with allogeneic MSCs from bone marrow (BM) $[16,17]$. MSCs from human umbilical cords (UC-MSCs) are bioequivalent to MSCs from bone marrow. In fact, UC-MSCs are genome stable and have lower immunogenicity and higher expansion ability compared with those from BM and other adult tissues $[18,19]$. UC-MSCs are also able to promote the production of Tregs in vitro [20] and to increase peripheral Treg in vivo [21]. In our previous studies, we demonstrated that UC-MSCs significantly increase the frequency of $\mathrm{CD} 4^{+} \mathrm{CD} 25^{\text {high }} \mathrm{CD} 45 \mathrm{RA}^{+}$Tregs and the production of antiinflammatory cytokines in co-cultures with PBMCs from healthy subjects [22]. In the current study, we further investigated the immunomodulatory effects of UC-MSCs on the frequency and immunosuppressive function of Tregs from the peripheral blood of MS patients by cocultures of UC-MSCs and PBMCs of MS patients.

\section{RESULTS}

\section{UC-MSCs significantly increased the frequency of Tregs among resting $\mathrm{CD4}^{+} \mathrm{T}$ cells derived from PBMCs of MS patients.}

We examined the frequency of CD4+CD25+CD127low/- Tregs in PBMCs from MS patients and healthy controls. No significant difference was detected for the frequency of CD4+CD25+CD127low/Tregs in CD4+ $\mathrm{T}$ cell sub-populations from MS compared to healthy controls. However, the frequency of CD4+CD25+CD127low/- Tregs in $\mathrm{CD}^{+} \mathrm{T}$ cells of MS patients was significantly increased in co-cultures with UC-MSCs compared to those without UC-MSCs (Figure 1D, $* * p<0.01$ ). Further examination showed that UCMSC co-cultures significantly increased the frequency of $\mathrm{CD} 4+\mathrm{CD} 25+\mathrm{CD} 127 \mathrm{low} /-$ Tregs not only in naïve PBMCs but also in PHA-activated PBMCs from MS (data not shown).

\section{UCMSC co-culture reversed the impaired suppressive function of Tregs from MS}

We evaluated the suppressive ability of CD4+CD25+CD127low/- Tregs from MS to the proliferation of $\mathrm{CD} 4+\mathrm{CD} 25-$ Teffs stimulated by PHA $(10 \mu \mathrm{g} / \mathrm{ml})$. Tregs from MS failed to inhibit the proliferation of PHA-stimulated allogeneic Teffs (Figure $2 \mathrm{~A}, \mathrm{p}<0.01)$ from heathy donors and autologous Teffs from the same MS patients (Figure $2 \mathrm{E}, \mathrm{p}<0.05$ ). These results demonstrated that suppressive functions are severely impaired in Tregs from MS. To investigate if UC-MSCs improve the impaired function of Tregs from MS, we co-cultured UC-MSCs with PBMCs from MS patients at a ratio of 1:5 for 3 days and then isolated $\mathrm{CD} 4+\mathrm{CD} 25+\mathrm{CD} 127 \mathrm{low} /-$ Tregs for functional examination. UC-MSC-primed CD4+CD25+CD127low/Tregs from MS significantly inhibited the proliferation of allogeneic CD4+CD25- Teffs from heathy donors (Figure $2 \mathrm{~A}$ ) and autologous CD4+CD25- Teffs from the same MS patients (Figure 2E). In fact, UCMSC-primed Tregs from MS reduced the proliferation index of PHA-stimulated allogeneic CD4+CD25- Teffs from heathy donors to the same degree as that produced by Tregs from heathy donors (Figure 2A); i.e., no significant difference was detected in the suppressive capacity of naïve Tregs from healthy donors and UCMSC-primed Tregs from MS patients, suggesting that UCMSC-co-cultures may have reversed 
the impaired suppressive function of Tregs from MS.

\section{UC-MSCs significantly improved regulatory cytokine production in co-cultures}

As shown in Figure 3, UCMSC co-cultures decreased the production of pro-inflammatory cytokine IFN- $\gamma$ (Figure 3D) but increased the production of the anti-inflammatory cytokines TGF- $\beta 1$, PGE2, and IL-10 (Figure 3A-3C) in PBMCs from MS. In fact, IL-10 was hardly detected in the supernatant of PBMC cultures from MS patients. However, co-cultures with UC-MSCs did not result in significant differences in the production of IL-10 in PBMC samples from healthy donors (data not shown).
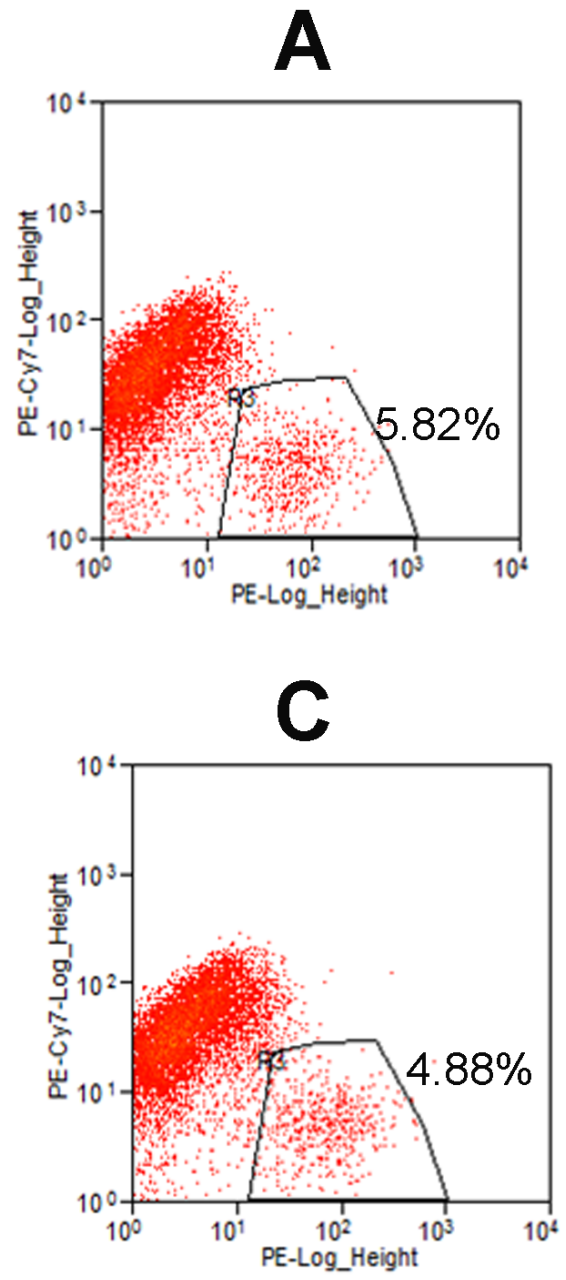

Figure 1: UC-MSCs significantly improved the frequency of Tregs among resting CD4 ${ }^{+}$T cells from MS. A, B, \& $\mathrm{C}$. Representative dot plot for $\mathrm{CD} 25^{+} \mathrm{CD} 127^{\text {low/- }}$ Tregs among resting CD4+ T cells in cultured PBMCs of healthy donors (A), MS patients (B) following 3-day co-culture without UC-MSCs and (C) with UC-MSCs (UC-MSCs:PBMCs=1:5). D. Bar graph showing that the frequency of $\mathrm{CD}^{+} \mathrm{CD} 25^{+} \mathrm{CD} 127^{\text {low/ }}$ Tregs among resting CD4+ T cells in PBMCs of MS was significantly increased following a 3-day co-culture with UC-MSCs compared to controls without UC-MSCs. No significant differences in the frequency of CD4 $4^{+} \mathrm{CD} 25^{+} \mathrm{CD} 127^{\text {low/- }} \mathrm{Tregs}$ were detected in naïve healthy donors and naïve MS patients. The data were expressed as mean $\pm \mathrm{SD}$ of four experiments. $* * p<0.01$. MS: multiple sclerosis, HC: healthy controls.

\section{UC-MSCs-priming enhanced the capacity of Tregs from MS to release IL-10}

We further investigated the possible mechanism(s) of the increased suppressive effect of Tregs from UCMSC-primed PBMCs of MS and measured IL-10 levels in the supernatant of Treg and Teff co-cultures by ELISA assay. IL-10 levels in the co-culture of Tregs from UCMSC-modulated PBMCs of MS patients and Teffs from either autologous or allogeneic origins were significantly increased compared to the co-cultures of Tregs from naïve PBMCs of MS without considering the source origins of Teffs (Figure 4). The improved capacity of Tregs for IL-10 release may have contributed to the increased suppressive
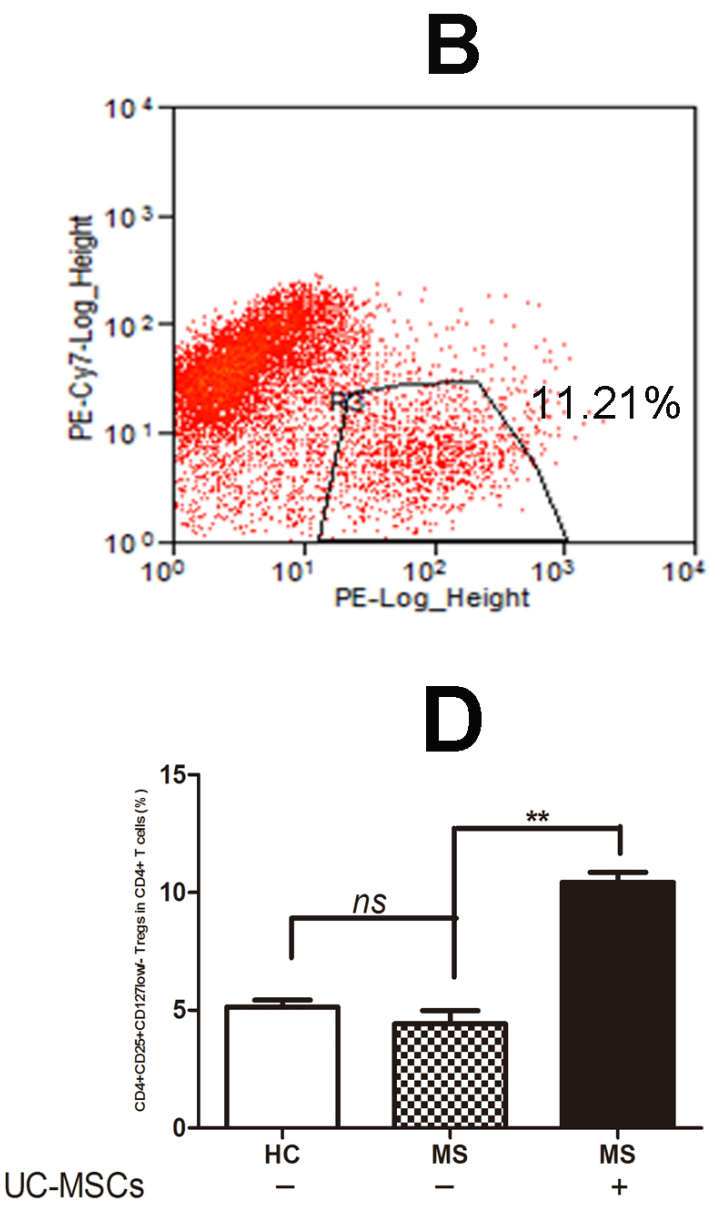

www.impactjournals.com/oncotarget 
function of Tregs from MS.

\section{DISCUSSION}

Tregs play a crucial role in homeostasis and self-tolerance control by dynamically suppressing the activation and expansion of autoreactive T-cells and other immune cells in peripheral and CNS systems [23-25]. The therapeutic potential of Tregs has been well documented in MS animal models [26, 27]. Clinical trials have demonstrated that systemic infusion of autologous Tregs in the treatment of autoimmune diseases such as type I
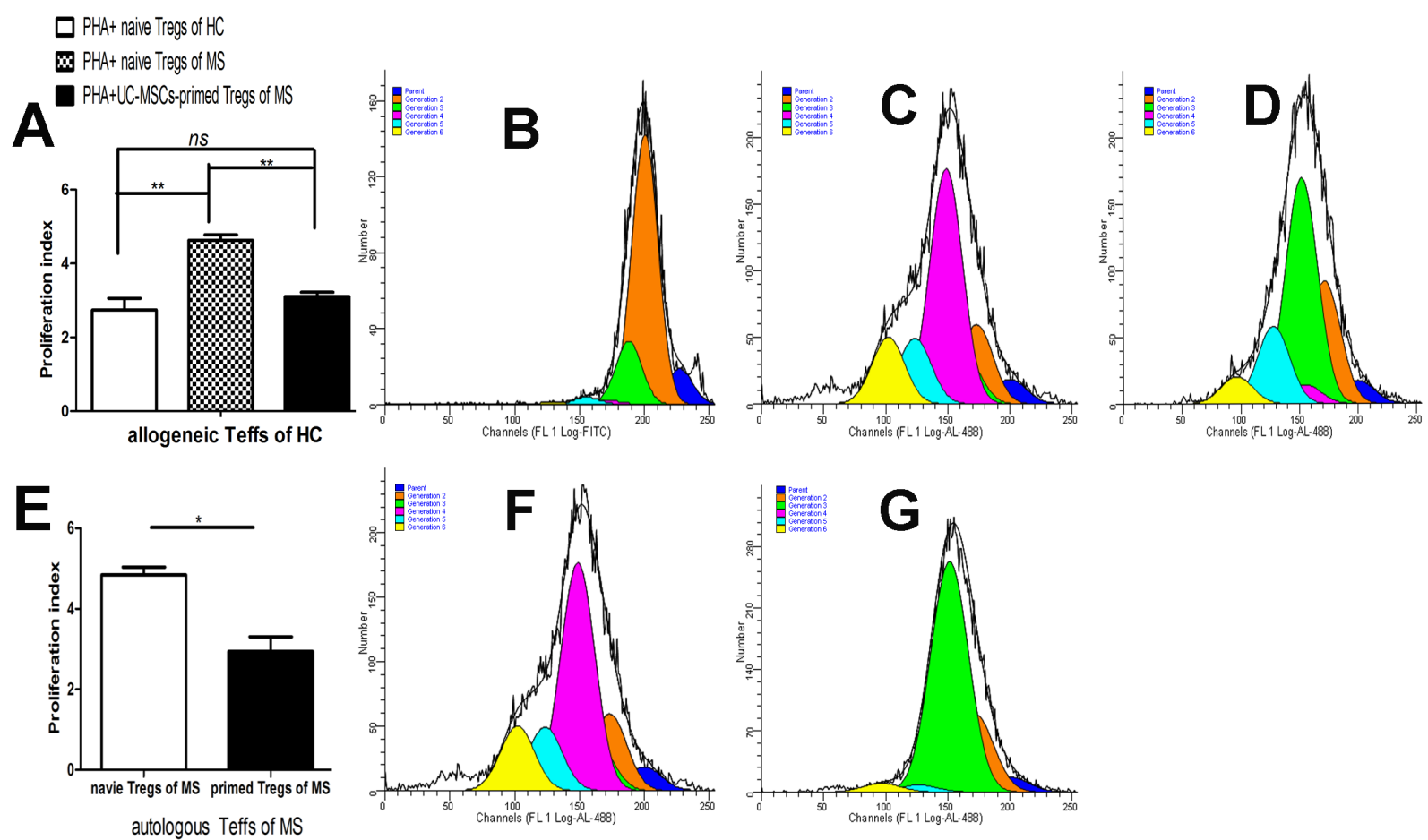

Figure 2: UC-MSCs reversed the impaired suppressive function of Tregs from MS. A. Bar graph showing that Tregs purified from naïve PBMCs (without UCMSC-priming) of MS patients failed to reduce the proliferation of allogeneic Teffs from healthy controls while Tregs purified from UCMSC-primed PBMCs of the same MS significantly reduced the proliferation index of allogeneic Teffs from healthy donors. No significant difference in the proliferation index of allogeneic Teffs was detected in Tregs purified from UCMSC-modulated PBMCs of MS and Tregs purified from naïve PBMCs of HC, suggesting that UC-MSC-priming may have reversed the functional deficits of Tregs from MS in the co-culture. Tregs:Teffs $=1: 10$. ${ }^{*} p<0.01,{ }^{*} p<0.05$. B, C, \& D. Representative ModFit results of CFSE-labeled and PHA-stimulated allogeneic CD4+CD25- Teffs of healthy donors in co-cultures with (B) Tregs sorted from naïve PBMCs of healthy donors, (C) Tregs sorted from naïve PBMCs of MS patients, and (D) Tregs sorted from UC-MSC-primed PBMCs of the same MS patients at a constant ratio of 1:10 for Tregs:Teffs. E. Bar graph showing that Tregs purified from UC-MSC-primed PBMCs of MS patients significantly reduced the proliferation index of autologous Teffs compared to Tregs purified from naïve PBMCs of the same MS patients. ${ }^{* *} p<0.01$. F \& G. Representative ModFit results of CFSE-labeled and PHA-stimulated autologous CD4 ${ }^{+} \mathrm{CD} 25^{-}$Teffs in cocultures with (F) Tregs purified from naïve PBMCs of MS patients and (G) Tregs purified from UC-MSC-primed PBMCs of the same MS patients at a ratio of 1:10 for Tregs:Teffs
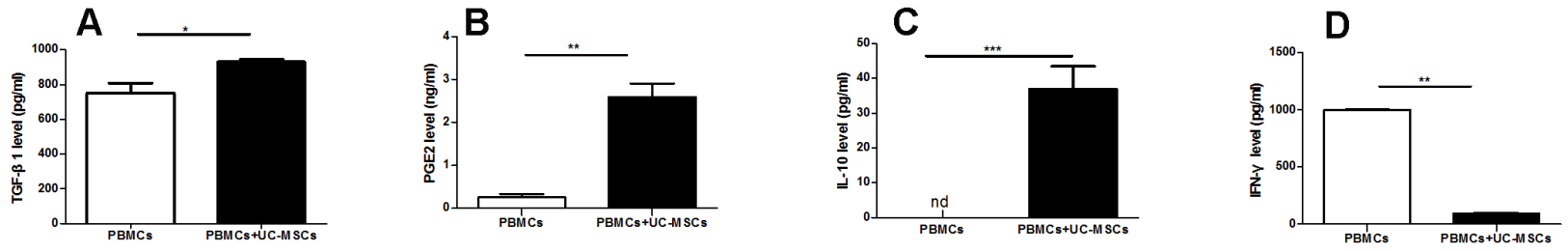

Figure 3: UC-MSCs significantly improved the production of cytokines in the co-cultures. Bar graph showing that UCMSCs significantly augmented the production of TGF- $\beta 1$ (A), PGE2 (B), and IL-10 (C) while reducing the production of IFN- $\gamma(\mathbf{D})$ in co-cultures of UC-MSCs and PBMCs from MS patients. Each experiment was performed in triplicate. Data were presented as mean \pm SD of four independent experiments. ${ }^{*} \mathrm{p}<0.05, * * \mathrm{p}<0.01, * * * \mathrm{p}<0.0001$. 
diabetes in children is well tolerated without major sideeffects [28]. However, a number of studies including our own identified multiple defects of Tregs in the peripheral blood of patients with MS and other autoimmune diseases; i.e. altered composition, reduced suppressive function, and poor secretion of inflammation-inhibiting cytokines $[4,12$, 30-33]. Although no difference was observed between the frequency of Tregs from MS patients and from healthy donors in our own studies (Figure 1D), our data revealed that Tregs from MS failed to suppress the proliferation of Teffs stimulated by in vitro PHA (Figure $2 \mathrm{~A} \& \mathrm{E}$ ). That is, autologous Treg from MS as a cell therapy is unlikely to be efficacious without a correction of the intrinsic impairments in Treg function.

MSCs are a subset of multipotent stem cells capable of self-renewing and differentiating into multiple mesenchymal cell lineages for connective tissues [2931 ], showing great potential in regenerative medicine. In addition, MSCs displayed multiple regulatory roles in the immune system by inducing the generation of Tregs via cell-cell contact between MSCs and T cells and secreting anti-inflammatory factors in vitro $[20,32]$, while in vivo studies showed that MSCs are able to control the progress of autoimmune diseases, including MS by inducing the expansion of Tregs [33]. UC-MSCs are isolated from discarded human umbilical cords, which offer an abundant and noninvasive source of MSCs without the concern of controversial ethical issues. UC-MSCs are viable alternatives to BM-derived MSCs. It has been reported that UC-MSCs share characteristics of BM-derived MSCs but are more primitive in nature with more powerful immune-modulating properties. For example, UC-MSCsuppressed mitogen induced lympho-proliferation to a greater extent than BM-derived MSCs. Furthermore, the extent of immune-suppression in inflammation is higher in UC-MSCs than in BM-derived MSCs [34]. In addition,
UC-MSCs are readily available, replicate rapidly in culture with a regular doubling time, and have low levels of senescence after repeated passages $[18,19]$.

To repair the defects of Tregs from MS patients, we co-cultured Tregs from MS patients with UC-MSCs and found UC-MSCs not only increased the frequency of Tregs of MS (Figure 1D) but also reversed their impaired suppressive function (Figure 2A). No significant difference in the suppressive function of Tregs was detected between Tregs from UC-MSC-primed PBMCs of MS patients and Tregs from naïve PBMCs from healthy donors, suggesting that the impaired suppressive function of Tregs may have reversed.

Some scientists suggest that Teffs resistance to the regulation of Tregs may also contribute to the pathogenesis of MS [35]. We did not examine whether there is a resistance of Teffs to Tregs in our current studies. However, our data showed that UC-MSC-primed Tregs from MS patients efficiently suppressed the proliferation of autologous Teffs stimulated by PHA compared to non-UC-MSC-primed Tregs from the same MS patients (Figure 2E).

As shown in Figure 3A-3C, we found PGE2, TGF- $\beta 1$, and IL-10 levels in the co-cultures of PBMCs with UC-MSCs were significantly higher than those in the cultures of PMBCs without UC-MSCs. In contrast, the levels of IFN- $\gamma$ in PMBC co-cultures with UC-MSCs were significantly lower than those of PBMC cultures without UC-MSCs (Figure 3D). Thus, soluble cytokines such as PGE2, TGF- $\beta 1$, IL- 10 , and IFN- $\gamma$ contribute to the phenotypic changes of mitogen-activated lymphocytes [36]. It is possible that UC-MSCs modulate the production of cytokines primarily through cell-cell contact because the conditioned medium of UC-MSCs only partially results in cytokine production alterations (data not shown). As suggested by other investigators [37], IL-10
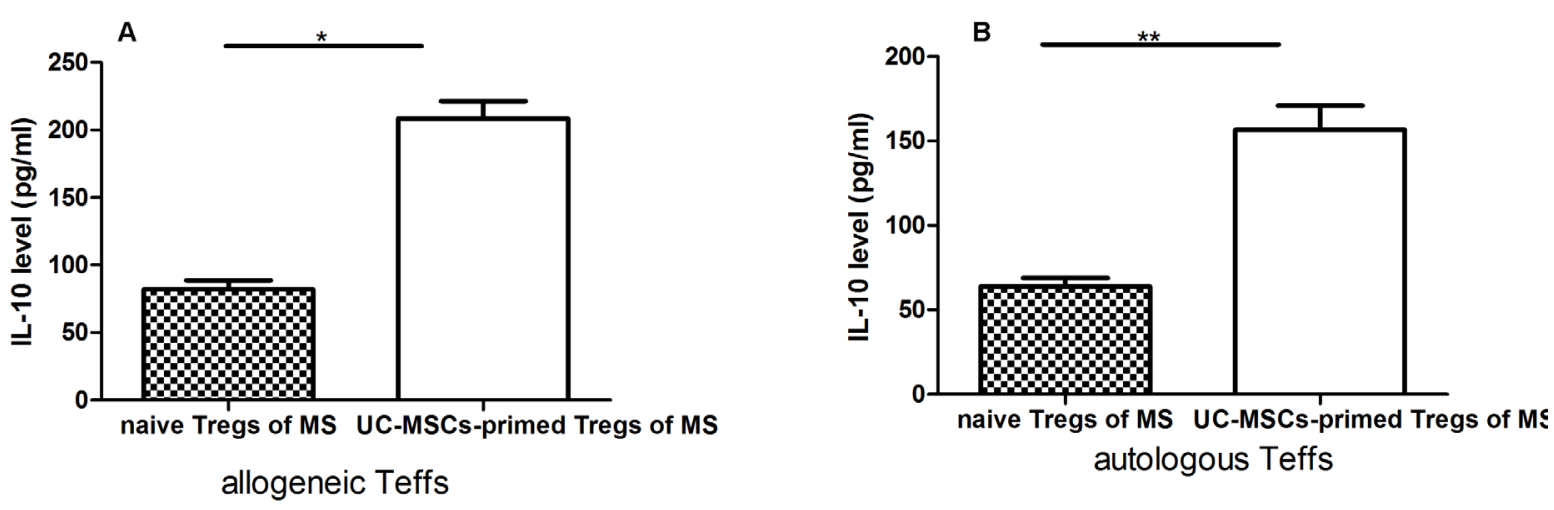

Figure 4: UC-MSC-primed Tregs from MS patients significantly increased IL-10 secretion levels in co-cultures with allogeneic Teffs from healthy donors or autologous Teffs from MS patients. A \& B: Bar graph showing that UC-MSCprimed Tregs from MS patients significantly increased IL-10 secretion levels in co-cultures with allogeneic Teffs (A) from healthy donors or autologous Teffs (B) from MS patients compared with naïve Tregs (without UC-MSC priming) from the same MS patients. Each experiment was performed in triplicate. Data were presented as mean $\pm \mathrm{SD}$ of four independent experiments. ${ }^{*} \mathrm{p}<0.05,{ }^{*} \mathrm{p}<0.01$. 
may be primarily responsible for the enhanced suppressive capability of MSC-exposed Tregs. Interestingly, we found that the levels of IL-10 in co-cultures of Tregs from UCMSC-primed PBMCs of MS patients with allogenic or autologous Teffs were significantly higher than those in the co-cultures of Tregs from naïve PBMCs of the same MS patients with allogenic or autologous Teffs (Figure 4 A\&B), suggesting that the improved cytokine production capacity may have contributed to the increased frequency and suppressive function of Tregs from MS. However, we did not verify which cell population secreted IL-10 into co-cultures because of blood sample limitations. Further studies are warranted to clarify this question.

In conclusion, we report for the first time that the intrinsic Treg defect in MS can be repaired in vitro using a UC-MSC-mediated immune modulation. UCMSC-primed Tregs from MS can effectively inhibit the proliferation of PHA-stimulated autologous Teffs from the same MS patients and allogeneic Teffs from healthy donors with a degree of inhibition observed in Tregs from healthy donors. Our studies provide valuable preliminary in vitro data to support the development of functionally normalized Tregs of autologous origins from individual patients with autoimmune diseases using a simple UC-MSC-based priming approach and may offer new therapeutic treatments for MS and other autoimmune diseases.

\section{MATERIALS AND METHODS}

\section{Isolation and culture of UC-MSCs}

Human umbilical cords $(n=3)$ were purchased from StemExpress (Placerville, CA, USA). We isolated and purified UC-MSCs according to the protocol previously published by our group [22]. In brief, the vessels of UC were removed to retain Wharton's jelly. Wharton's jelly tissue was then cut into $1 \mathrm{~mm}^{3}$ pieces, added to the bottom of the tissue culture dish (BD, Franklin Lakes, New Jersey), and incubated at $37^{\circ} \mathrm{C}$ in a $5 \%$ carbon dioxide incubator without culture medium for two hours to allow tissue attachment. $15 \mathrm{ml}$ complete culture medium containing DMEM (low glucose) supplemented with $10 \%$ FBS (fetal bovine serum), 1\% L-glutamine, and $1 \%$ penicillin-streptomycin (all from Invitrogen, Grand Island, NY) was added to the tissue culture dishes and incubated for an additional 7 days at $37^{\circ} \mathrm{C}$ and $5 \%$ carbon dioxide incubator. The purified Wharton's jelly pieces were removed and the primary cells that had migrated out of the Wharton's jelly tissues and attached to tissue culture dishes were passaged by a 1 -min treatment with $0.25 \%$ trypsin and $0.02 \%$ EDTA at $37^{\circ} \mathrm{C}$. The medium was changed every 3 days. UC-MSCs were further passaged when they reached 90 confluency. All UC-MSCs used in experiments were passages 3-5.

\section{Co-cultures of UC-MSCs and PBMCs}

PBMCs obtained from patients with relapsingremitting multiple sclerosis (RRMS, $\mathrm{n}=12$, female $=8$, male $=4$ ), mean age 53.75 years old ( 49 years to 64 years old) and healthy donors $(\mathrm{n}=10$, female $=4$, male $=6)$, mean age 28.38 years old ( 22 years to 39 years old) were purchased from AllCells Inc (Emeryville, CA) or StemExpress (Placerville, CA). All patients were under treatment; one with Copaxone, one with Tysabri, one with Avonex, and one with HCTZ. The others were under treatment with unknown drugs. The duration of the disease and prior treatment history were unknown. Before co-culture, allogenic UC-MSCs $\left(2 \times 10^{5}\right)$ were seeded into each well of a 6-well tissue culture plate (BD), cultured in $2 \mathrm{ml}$ of advanced RPMI 1640 culture medium (Invitrogen, Grand Island, NY, USA) supplemented with $10 \%$ FBS, $2 \mathrm{mM}$ glutamine, $100 \mathrm{U} / \mathrm{ml}$ penicillin and streptomycin, and allowed to adhere at $37^{\circ} \mathrm{C}$ and $5 \%$ carbon dioxide incubator for $4 \mathrm{~h}$. PBMCs $\left(1 \times 10^{6}\right)$ from MS patients and healthy donors were directly loaded onto the cultured UC-MSCs and co-cultured for $72 \mathrm{~h}$ in a culture medium (2ml/well) containing advanced RPMI 1640 (Invitrogen, Grand Island, NY, USA) supplemented with $10 \%$ FBS (fetal bovine serum, Invitrogen), 1\% L-glutamine, and $1 \%$ penicillin-streptomycin (Invitrogen, Grand Island, NY). PBMCs cultured alone (without UC-MSCs) served as controls. Each experiment was performed in triplicate. After 3 days co-culture, the suspended PBMCs were collected to perform flow analysis for $\mathrm{CD} 4{ }^{+} \mathrm{CD} 25^{+} \mathrm{CD} 127^{\text {low/ } /}$ Tregs frequency and cell sorting for purified $\mathrm{CD} 4{ }^{+} \mathrm{CD} 25^{+} \mathrm{CD} 127^{\text {low/ }}$ Tregs and $\mathrm{CD} 4{ }^{+} \mathrm{CD} 25^{-}$ $\mathrm{T}$ effectors (Teff). The cell-free supernatant was collected by centrifugation (1000 rpm x $5 \mathrm{~min}$ ) and kept frozen at $-80^{\circ} \mathrm{C}$ until ELISA analysis.

\section{Flow cytometry and $\mathrm{CD}^{+} \mathrm{CD25}^{-}$Teffs and $\mathrm{CD}^{+} \mathrm{CD}^{+}{ }^{+} \mathrm{CD} 127^{\text {low/ }}$ Tregs purification}

Flow cytometry analysis was performed according to our previous protocol [22] . In brief, $1 \times 10^{6}$ suspended UC-MSC-primed or naïve PBMCs from MS patients or naïve PBMCs from healthy donors were washed with washing buffer (PBS containing 2\% FBS) and incubated in $100 \mu \mathrm{l}$ washing buffer supplement with $10 \mu \mathrm{l}$ various fluorescent dye-conjugated specific antibodies (APCconjugated mouse anti-human CD4, PE-conjugated mouse anti-human CD25, and PE-Cy7-conjugated mouse anti-human CD127) on ice for 30min. All antibodies were purchased from eBioscience Inc (USA). After incubation, $2 \times 10^{5}$ suspended naïve or UC-MSC-primed PBMCs from MS patients or naïve PBMCs from healthy donors were washed twice with washing buffer, resuspended in $200 \mu \mathrm{l}$ 
washing buffer, and analyzed by the LSRFortessa ${ }^{\mathrm{TM}}$ cell analyzer (BD, Franklin Lakes, New Jersey). The left $8 \times 10^{5}$ suspended naïve or UC-MSC-primed PBMCs from MS patients or naïve PBMCs from healthy donors were used to purify $\mathrm{CD}^{+} \mathrm{CD} 25^{+} \mathrm{CD} 127^{\text {low/- }}$ Tregs and $\mathrm{CD} 4^{+} \mathrm{CD} 25^{-}$ $\mathrm{T}$ effectors (Teff). Purification of $\mathrm{CD}^{+} \mathrm{CD} 25^{-}$Teffs and $\mathrm{CD} 4{ }^{+} \mathrm{CD} 25^{+} \mathrm{CD} 127^{\text {low/- }}$ Tregs was performed according to the protocol of Zhao et al with minor modifications [8]. $8 \times 10^{5}$ stained UC-MSC-primed or naïve PBMCs from MS patients or naïve PBMCs from healthy donors were resuspended in $200 \mu \mathrm{l}$ buffer containing PBS, 2\% BSA (bovine serum albumin), and $0.02 \%$ EDTA. Separation of CD $4^{+} \mathrm{CD} 25^{-}$Teffs and $\mathrm{CD} 4^{+} \mathrm{CD} 25^{+} \mathrm{CD} 127^{\text {low/- }}$ Tregs was conducted with a Dako-Cyomation MoFlo high speed sorter (Dako, Houston, TX).

\section{Cell proliferation assay}

Purified CD4 ${ }^{+} \mathrm{CD} 25^{-}$Teffs from PBMCs of healthy donors or MS patients were labeled with $10 \mu \mathrm{M}$ CFSE (5,6-carboxyfluorescein diacetate succinimidyl ester, Invitrogen) in pre-warmed PBS containing 0.1\% BSA at a final concentration of $1 \times 10^{6}$ cells $/ \mathrm{ml}$ for $15 \mathrm{~min}$ at $37^{\circ} \mathrm{C}$ in the dark, a protocol described by our group[22]. After incubation, CFSE-labeled CD4 ${ }^{+} \mathrm{CD} 25^{-}$Teffs were washed twice using 5 volumes of ice-cold culture media (advanced RPMI 1640 supplemented with 10\% FBS, 1\% L-glutamine, and $1 \%$ penicillin-streptomycin). $1 \times 10^{4}$ $\mathrm{CD} 4{ }^{+} \mathrm{CD} 25^{+} \mathrm{CD} 127^{\text {low/- }}$ Tregs purified from UC-MSCprimed or naïve PBMCs of MS patients or healthy donors were co-cultured with $1 \times 10^{5}$ CFSE-labeled CD4 ${ }^{+} \mathrm{CD} 25$ Teffs (Tregs:Teffs $=1: 10$ in $0.2 \mathrm{ml}$ ) in the presence of PHA $(10 \mu \mathrm{g} / \mathrm{ml})$. Five groups were included in this experiment: Tregs from naïve PBMCs of healthy donors + Teffs from naïve PBMCs of healthy donors; Tregs from naïve PBMCs of MS patients + Teffs from naïve PBMCs of healthy donors; Tregs from UC-MSC-primed PBMCs of MS patients + Teffs from naïve PBMCs of healthy donors; Tregs from naïve PBMCs of MS patients + Teffs from naïve PBMCs of MS patients; Tregs from UCMSC-primed PBMCs of MS patients + Teffs from naïve PBMCs of MS patients. Each experiment was performed in triplicate. Three days later, cells were harvested for flow cytometry by LSRFortessa ${ }^{\mathrm{TM}}$ cell analyzer (BD, Franklin Lakes, New Jersey) at 488nm excitation. The proliferation index was calculated using ModFit Software (Verity Software House). Cell-free supernatant was collected by centrifugation (1000 rpm x 5min) and kept frozen at $-80^{\circ} \mathrm{C}$ until ELISA analysis.

\section{Cytokine analysis by ELISA}

Cell-free supernatant was collected after co-culture of PBMCs with UC-MSCs (1:5) and cultures of PBMCs without UC-MSCs for 3 days and used to measure the concentrations of cytokine prostaglandin E2 (PGE2), transforming growth factor (TGF)- $\beta 1$, interleukin-10 (IL10 ), and interferon (IFN) $-\gamma$ by ELISA analysis according to the manufacturer's protocol. Cell-free supernatant collected after Tregs and Teffs (1:10) co-cultures for 3 days were used to measure the concentration of cytokine IL-10 by ELISA analysis according to the manufacturer's protocol. TGF- $\beta 1$, IL-10, and IFN- $\gamma$ ELISA kits were purchased from eBioscience (San Diego, CA), and the PGE2 ELISA kit was purchased from R\&D System (Minneapolis, CA). The collected cell-free supernatant was placed in a 96-well specific antibody-coated plate. Absorbance was recorded at $450 \mathrm{~nm}$ using a microplate reader (Bio-Rad, Philadelphia, PA). Each cytokine standard and sample was run in duplicate.

\section{Statistical analysis}

Statistical analysis was performed using GraphPad Prism 5 (GraphPad). Data were expressed as means \pm SEM. A two-sample T test or one-way ANOVA was used for statistical analysis. A p-value $<0.05$ was considered significant.

\section{ACKNOWLEDGMENTS}

This research is supported by grants of 1) DoD (PR100499P1), 2) Boothroyd Foundation, USA, 3) Program of 5150 in Jinan city, and 4) Taishan Oversea Scholars in Shandong province, China to T. QU.

\section{CONFLICTS OF INTEREST}

The authors declare no conflicts of interest.

\section{REFERENCES}

1. Kostic M, Stojanovic I, Marjanovic G, Zivkovic N, Cvetanovic A. Deleterious versus protective autoimmunity in multiple sclerosis. Cellular immunology. 2015; 296: 12232. doi: 10.1016/j.cellimm.2015.04.006.

2. Tullman MJ. Overview of the epidemiology, diagnosis, and disease progression associated with multiple sclerosis. The American journal of managed care. 2013; 19: S15-20.

3. Kumar M, Putzki N, Limmroth V, Remus R, Lindemann M, Knop D, Mueller N, Hardt C, Kreuzfelder E, Grosse-Wilde H. CD4+CD25+FoxP3+ T lymphocytes fail to suppress myelin basic protein-induced proliferation in patients with multiple sclerosis. Journal of neuroimmunology. 2006; 180: 178-84. doi: 10.1016/j.jneuroim.2006.08.003.

4. Huan J, Culbertson N, Spencer L, Bartholomew R, Burrows GG, Chou YK, Bourdette D, Ziegler SF, Offner H, Vandenbark AA. Decreased FOXP3 levels in multiple sclerosis patients. Journal of neuroscience research. 2005; 
81: 45-52. doi: 10.1002/jnr.20522.

5. Dalla Libera D, Di Mitri D, Bergami A, Centonze D, Gasperini C, Grasso MG, Galgani S, Martinelli V, Comi G, Avolio C, Martino G, Borsellino G, Sallusto F, et al. T regulatory cells are markers of disease activity in multiple sclerosis patients. PloS one. 2011; 6: e21386. doi: 10.1371/ journal.pone.0021386.

6. Sakaguchi S, Wing K, Onishi Y, Prieto-Martin P, Yamaguchi T. Regulatory T cells: how do they suppress immune responses? International Immunology. 2009; 21: 1105-11. doi: 10.1093/intimm/dxp095.

7. Lim JY, Park MJ, Im KI, Kim N, Jeon EJ, Kim EJ, Cho ML, Cho SG. Combination cell therapy using mesenchymal stem cells and regulatory T-cells provides a synergistic immunomodulatory effect associated with reciprocal regulation of TH1/TH2 and th17/treg cells in a murine acute graft-versus-host disease model. Cell transplantation. 2014; 23: 703-14. doi: 10.3727/096368913X664577.

8. Zhao Y, Lin B, Darflinger R, Zhang Y, Holterman MJ, Skidgel RA. Human cord blood stem cell-modulated regulatory $\mathrm{T}$ lymphocytes reverse the autoimmune-caused type 1 diabetes in nonobese diabetic (NOD) mice. PloS one. 2009; 4: e4226. doi: 10.1371/journal.pone.0004226.

9. Zhang D, Zhang W, Ng TW, Wang Y, Liu Q, Gorantla $\mathrm{V}$, Lakkis F, Zheng XX. Adoptive cell therapy using antigen-specific CD4(-)CD8(-)T regulatory cells to prevent autoimmune diabetes and promote islet allograft survival in NOD mice. Diabetologia. 2011; 54: 2082-92. doi: 10.1007/ s00125-011-2179-4.

10. Thome R, Moraes AS, Bombeiro AL, Farias Ados S, Francelin C, da Costa TA, Di Gangi R, dos Santos LM, de Oliveira AL, Verinaud L. Chloroquine treatment enhances regulatory $\mathrm{T}$ cells and reduces the severity of experimental autoimmune encephalomyelitis. PloS one. 2013; 8: e65913. doi: 10.1371/journal.pone.0065913.

11. Venken K, Hellings N, Thewissen M, Somers V, Hensen K, Rummens JL, Medaer R, Hupperts R, Stinissen P. Compromised CD4+ CD25(high) regulatory T-cell function in patients with relapsing-remitting multiple sclerosis is correlated with a reduced frequency of FOXP3-positive cells and reduced FOXP3 expression at the single-cell level. Immunology. 2008; 123: 79-89. doi: IMM2690 [pii] 10.1111/j.1365-2567.2007.02690.x.

12. Viglietta V, Baecher-Allan C, Weiner HL, Hafler DA. Loss of functional suppression by $\mathrm{CD} 4+\mathrm{CD} 25+$ regulatory $\mathrm{T}$ cells in patients with multiple sclerosis. J Exp Med. 2004; 199: 971-9. doi: 10.1084/jem.20031579 jem.20031579 [pii].

13. English K, Ryan JM, Tobin L, Murphy MJ, Barry FP, Mahon BP. Cell contact, prostaglandin E(2) and transforming growth factor beta 1 play non-redundant roles in human mesenchymal stem cell induction of CD4+CD25(High) forkhead box P3+ regulatory T cells. Clinical and experimental immunology. 2009; 156: 149-60. doi: $10.1111 / \mathrm{j} .1365-2249.2009 .03874 . x$.
14. Boumaza I, Srinivasan S, Witt WT, Feghali-Bostwick C, Dai Y, Garcia-Ocana A, Feili-Hariri M. Autologous bone marrow-derived rat mesenchymal stem cells promote PDX1 and insulin expression in the islets, alter T cell cytokine pattern and preserve regulatory $\mathrm{T}$ cells in the periphery and induce sustained normoglycemia. Journal of autoimmunity. 2009; 32: 33-42. doi: 10.1016/j.jaut.2008.10.004.

15. Zhu J, Zhang J, Li Q, Du Y, Qiao B, Hu X. Transplanting of mesenchymal stem cells may affect proliferation and function of $\mathrm{CD} 4(+) \mathrm{T}$ cells in experimental autoimmune encephalomyelitis. Experimental and clinical transplantation. 2012; 10: 492-500. doi: 10.6002/ ect.2011.0197.

16. Yan Z, Zhuansun Y, Chen R, Li J, Ran P. Immunomodulation of mesenchymal stromal cells on regulatory $\mathrm{T}$ cells and its possible mechanism. Experimental cell research. 2014; 324: 65-74. doi: 10.1016/j. yexcr.2014.03.013.

17. Liu Q, Zheng H, Chen X, Peng Y, Huang W, Li X, Li G, Xia W, Sun Q, Peng Xiang A. Human mesenchymal stromal cells enhance the immunomodulatory function of CD8CD28 regulatory T cells. Cellular \& molecular immunology. 2014. doi: 10.1038/cmi.2014.118.

18. Zhang H, Zhang B, Tao Y, Cheng M, Hu J, Xu M, Chen H. Isolation and characterization of mesenchymal stem cells from whole human umbilical cord applying a single enzyme approach. Cell biochemistry and function. 2012; 30: 643-9. doi: $10.1002 / \mathrm{cbf} .2843$.

19. Troyer DL, Weiss ML. Wharton's jelly-derived cells are a primitive stromal cell population. Stem cells. 2008; 26: 591-9. doi: 2007-0439 [pii] 10.1634/stemcells.2007-0439.

20. Tipnis S, Viswanathan C, Majumdar AS. Immunosuppressive properties of human umbilical cordderived mesenchymal stem cells: role of B7-H1 and IDO. Immunology and Cell Biology. 2010; 88: 795-806. doi: 10.1038/icb.2010.47.

21. Sun L, Wang D, Liang J, Zhang $H$, Feng $X$, Wang $H$, Hua B, Liu B, Ye S, Hu X, Xu W, Zeng X, Hou Y, et al. Umbilical cord mesenchymal stem cell transplantation in severe and refractory systemic lupus erythematosus. Arthritis and rheumatism. 2010; 62: 2467-75. doi: 10.1002/ art.27548.

22. Yang H, Xie Z, Wei L, Bi J. Systemic Transplantation of Human Umbilical Cord Derived Mesenchymal Stem Cells-Educated T Regulatory Cells Improved the Impaired Cognition in AbetaPPswe/PS1dE9 Transgenic Mice. PloS one. 2013; 8: e69129. doi: 10.1371/journal.pone.0069129 PONE-D-13-11592 [pii].

23. Kim JM, Rasmussen JP, Rudensky AY. Regulatory T cells prevent catastrophic autoimmunity throughout the lifespan of mice. Nat Immunol. 2007; 8: 191-7. doi: ni1428 [pii] 10.1038/ni1428.

24. Sakaguchi S, Yamaguchi T, Nomura T, Ono M. Regulatory T cells and immune tolerance. Cell. 2008; 133: 775-87. doi: 10.1016/j.cell.2008.05.009S0092-8674(08)00624-7 [pii]. 
25. Sakaguchi S, Ono M, Setoguchi R, Yagi H, Hori S, Fehervari Z, Shimizu J, Takahashi T, Nomura T. Foxp3+ $\mathrm{CD} 25+\mathrm{CD} 4+$ natural regulatory $\mathrm{T}$ cells in dominant self-tolerance and autoimmune disease. Immunol Rev. 2006; 212: 8-27. doi: IMR427 [pii]10.1111/j.01052896.2006.00427.x.

26. Kohm AP, Carpentier PA, Anger HA, Miller SD. Cutting edge: $\mathrm{CD} 4+\mathrm{CD} 25+$ regulatory $\mathrm{T}$ cells suppress antigenspecific autoreactive immune responses and central nervous system inflammation during active experimental autoimmune encephalomyelitis. J Immunol. 2002; 169: 4712-6. doi:

27. Anderton SM, Liblau RS. Regulatory T cells in the control of inflammatory demyelinating diseases of the central nervous system. Curr Opin Neurol. 2008; 21: 248-54. doi: 10.1097/ WCO.0b013e3282febf5800019052-200806000-00006 [pii].

28. Marek-Trzonkowska N, Mysliwiec M, Dobyszuk A, Grabowska M, Techmanska I, Juscinska J, Wujtewicz MA, Witkowski P, Mlynarski W, Balcerska A, Mysliwska J, Trzonkowski P. Administration of CD4+CD25highCD127regulatory $\mathrm{T}$ cells preserves beta-cell function in type 1 diabetes in children. Diabetes Care. 2012; 35: 1817-20. doi: 10.2337/dc12-0038dc12-0038 [pii].

29. Majumdar MK, Thiede MA, Mosca JD, Moorman M, Gerson SL. Phenotypic and functional comparison of cultures of marrow-derived mesenchymal stem cells (MSCs) and stromal cells. J Cell Physiol. 1998; 176: 57-66. doi: 10.1002/(SICI)1097-4652(199807)176:1<57::AIDJCP7>3.0.CO;2-7 [pii]10.1002/(SICI)10974652(199807)176:1<57::AID-JCP7>3.0.CO;2-7.

30. Pereira RF, Halford KW, O'Hara MD, Leeper DB, Sokolov BP, Pollard MD, Bagasra O, Prockop DJ. Cultured adherent cells from marrow can serve as long-lasting precursor cells for bone, cartilage, and lung in irradiated mice. Proceedings of the National Academy of Sciences of the United States of America. 1995; 92: 4857-61.

31. Pittenger MF, Mackay AM, Beck SC, Jaiswal RK, Douglas R, Mosca JD, Moorman MA, Simonetti DW, Craig S, Marshak DR. Multilineage potential of adult human mesenchymal stem cells. Science. 1999; 284: 143-7.
32. Ghannam S, Pene J, Torcy-Moquet G, Jorgensen C, Yssel H. Mesenchymal Stem Cells Inhibit Human Th17 Cell Differentiation and Function and Induce a T Regulatory Cell Phenotype. The Journal of Immunology. 2010; 185: 302-12. doi: 10.4049/jimmunol.0902007.

33. Yousefi F, Ebtekar M, Soleimani M, Soudi S, Hashemi SM. Comparison of in vivo immunomodulatory effects of intravenous and intraperitoneal administration of adiposetissue mesenchymal stem cells in experimental autoimmune encephalomyelitis (EAE). Int Immunopharmacol. 2013; 17: 608-16. doi: 10.1016/j.intimp.2013.07.01 6S1567-5769(13)00301-9 [pii].

34. Prasanna SJ, Gopalakrishnan D, Shankar SR, Vasandan AB. Pro-inflammatory cytokines, IFNgamma and TNFalpha, influence immune properties of human bone marrow and Wharton jelly mesenchymal stem cells differentially. PloS one. 2010; 5: e9016. doi: 10.1371/journal.pone.0009016.

35. Schneider A, Long SA, Cerosaletti K, Ni CT, Samuels P, Kita M, Buckner JH. In active relapsing-remitting multiple sclerosis, effector $\mathrm{T}$ cell resistance to adaptive $\mathrm{T}$ (regs) involves IL-6-mediated signaling. Sci Transl Med. 2013; 5: 170ra15. doi: 10.1126/scitranslmed.30049705/170/170ra15 [pii].

36. Ghannam S, Bouffi C, Djouad F, Jorgensen C, Noel D. Immunosuppression by mesenchymal stem cells: mechanisms and clinical applications. Stem Cell Res Ther. 2010; 1: 2. doi: 10.1186/scrt2scrt2 [pii].

37. Yan Z, Zhuansun Y, Chen R, Li J, Ran P. Immunomodulation of mesenchymal stromal cells on regulatory $\mathrm{T}$ cells and its possible mechanism. Exp Cell Res. 2014; 324: 65-74. doi: 10.1016/j.yexcr.2014.03.013 S0014-4827(14)00129-3 [pii]. 